\title{
Economic Analysis of Intravenous vs. Subcutaneously Administered Trastuzumab for the Treatment of HER2+ Early Breast Cancer in Malaysia
}

\author{
Wei Ching Lee', Mohd Roslan Haron², Kong Leong Yuㄹ, Flora Li Tze Chong, Adrian Goh5, \\ Soraya Azmi ${ }^{*}$ \\ ${ }^{1}$ Radiotherapy and Oncology Department, Penang General Hospital, Georgetown, Malaysia \\ ${ }^{2}$ Radiotherapy and Oncology Department, Sultan Ismail Hospital, Johor Bahru, Malaysia \\ ${ }^{3}$ Oncology \& Palliative Care Department, Sarawak General Hospital, Kuching, Malaysia \\ ${ }^{4}$ Radiotherapy \& Oncology Department, Likas Hospital, Kota Kinabalu, Malaysia \\ ${ }^{5}$ Veras Research, Petaling Jaya, Malaysia \\ Email: "soraya.azmi@verasresearch.com
}

Received 19 November 2015; accepted 8 January 2016; published 11 January 2016

Copyright (C) 2016 by authors and Scientific Research Publishing Inc.

This work is licensed under the Creative Commons Attribution International License (CC BY). http://creativecommons.org/licenses/by/4.0/

(c) ())

\section{Abstract}

Human epidermal growth factor receptor-2 over expression has been reported in $23 \%-37.3 \%$ of Malaysian breast cancer patients. Trastuzumab is a recommended adjuvant therapy for patients with early breast cancer. Currently, trastuzumab is administered intravenously but a new subcutaneous formulation could save time and costs. In Malaysia, the Ministry of Health funds most of the costs of secondary care, including cancer treatment. We performed a cost-minimisation analysis from the Ministry of Health and societal perspectives by adapting an excel-based mathematical model. Model inputs comprised of drug, consumables and personnel costs. Data sources were government statistics, price lists, government salary scales and estimated personnel time costs. Doctors, nurses and pharmacists at four hospitals estimated the time to perform necessary tasks following their usual practice. Results showed that subcutaneous trastuzumab could, on average, save an estimated 43.5 minutes of pharmacists' time and 11.6 minutes and 4.9 minutes of nurses' time administering loading dose and subsequent doses, respectively. Total cost savings were RM7561 (8.7\%) per patient per year, consisting of RM6496 (7.6\%) reduction in drug cost and RM1065 (66.2\%) reduction in non-drug costs. Similarly, cost savings from the societal perspective were RM7820 per patient per year, comprising RM6496 (7.6\%) and RM1325 (63.7\%) of drug and non-drug costs savings, respectively. Sensitivity analyses showed that cost savings on subcutaneous formulation ranged from RM3715 to RM11408 to the Ministry of Health. Overall, subcutaneous trastuzumab is estimated

\footnotetext{
${ }^{*}$ Corresponding author.
}

How to cite this paper: Lee, W.C., Haron, M.R., Yu, K.L., Chong, F.L.T., Goh, A. and Azmi, S. (2016) Economic Analysis of Intravenous vs. Subcutaneously Administered Trastuzumab for the Treatment of HER2+ Early Breast Cancer in Malaysia. Advances in Breast Cancer Research, 5, 1-13. http://dx.doi.org/10.4236/abcr.2016.51001 
to be cost-saving from Ministry of Health and societal perspectives. Other benefits from saving patients' and carers' time and convenience could not be taken into account. This study was a first to investigate cost-savings from subcutaneous trastuzumab in a middle-income Asian country and the findings were consistent with findings of earlier studies. Given the favourable impact on time and costs, subcutaneous trastuzumab should be considered a viable option for Malaysian patients.

\section{Keywords}

Cost-Minimisation Analysis, Trastuzumab, Subcutaneous, Intravenous, Breast Cancer, Malaysia

\section{Introduction}

The impact of breast cancer on women in Asia is significant. It is the most common type of cancer among women in the Asia-Pacific region accounting for 18\% of all cases in 2012 [1]. In Malaysia, breast cancer has an age-standardised incidence rate of 38.7 per 100,000 population, which is higher than the average incidence for the rest of South-Eastern Asia at 34.8 per 100,000 population [1]. Furthermore, along with Thailand, Malaysia is experiencing increased breast cancer mortality rates compared to other nations in the Asia-Pacific region. Malaysia had recorded an increase of 6\% per year between 1997 and 2008 comparable to the increase in Thailand which was reported as 7\% per year from 2000 to 2006 [1]. Breast cancer is also the leading cause of cancer-related deaths in women in Malaysia at 25\% of all cancer related deaths [1]. In general, the reasons for the high rate of mortality are likely to be multifactorial and include social and economic factors such as a lack of understanding of the disease, lack of education and awareness, inadequate diagnostic equipment and facilities, competing health care needs as well as a reliance on traditional remedies [1]-[3]. While Malaysian incidence rates seem to not have increased as rapidly as mortality rates, early detection and efforts to increase awareness and prevent occurrence should continue to be a focus. Nonetheless, efforts to increase access to diagnosis and provision of effective treatment should also not lag behind in the overall strategy [4].

Few would argue that cancer treatment is costly, thus any measures that could save costs should be considered. In Malaysia, most of this care is supported by the government through a highly subsidized healthcare system [5] [6]. Primary outpatient care tends to be sought and provided in the private setting, at $62 \%$ versus $38 \%$ in government facilities. On the other hand, secondary care is largely borne by the government with $83 \%$ of care provided by government facilities compared to $17 \%$ sought and provided by private facilities [5]. Few studies have tried to address this issue with respect to Malaysia. An unpublished paper by Das and Win, estimated that the cost of breast cancer treatment was between RM10000 and 30,000 per patient [7]. The paper did not state what was included in this estimate nor the methodology that was used to estimate the cost. A more detailed study by Yen and colleagues investigated the impact of breast cancer treatment from the patient's perspective [8]. Patients included in the study were both those obtained treatment from government as well as private facilities and those with and without insurance. The authors estimated that patients could incur expenses of up to RM90000 to obtain treatment. It is important to bear in mind that these are fees charged to patients and do not reflect the economic cost of treatment hence it was possible for patients to pay very little for their treatment which is a reflection of the generous subsidies afforded by the government healthcare system. Hence taking into consideration the government's perspective tends to be important in a Malaysian study that investigates the economic costs of any chronic or catastrophic illnesses.

Trastuzumab (Herceptin) is a monoclonal antibody that targets HER2 positive breast cancer and its efficacy has been well-established through clinical studies [9] [10]. Studies conducted in Western countries have also shown a positive cost-utility impact of trastuzumab when Quality-Adjusted Life Years (QALYs) are taken into account [11]-[13]. Within the region, a study performed in Singapore also found that trastuzumab would be likely to generate net societal benefits over costs which would make it cost-effective for Singapore [14]. Over expression of the human epidermal growth factor receptor 2 (HER2) is associated with poor prognosis in breast cancer and has been reported in 23\% to $37.3 \%$ of Malaysian breast cancer patients [15]. According to a study among 3557 cases of breast cancer in Malaysia between 1994 and 2008, the proportion of HER+ breast cancers increased by $2 \%$ every 5 years [2]. While trastuzumab is used in both the private and public settings in the coun- 
try, its use in the public setting is dependent on individual clinical need and its affordability at the treating hospital. The established method of administration is intravenous (IV). IV trastuzumab is administered through venous access at an initial dose of $8 \mathrm{mg} / \mathrm{kg}$ over 90 minutes then $6 \mathrm{mg} / \mathrm{kg}$ over $30-90$ minutes for subsequent doses [16]-[18].

On the other hand, a new subcutaneous (SC) formulation has been approved by the European Medicines Agency for HER2+ breast cancer in June 2013 [19]. The SC formulation trastuzumab is administered in a fixed dose $600 \mathrm{mg}$ injection (i.e. independent of patient weight) from a hand-held syringe or single-injection device (SID) over a duration of five minutes [16] [20]. The efficacy of SC trastuzumab has been reported in the HannaH clinical trial which showed that the SC formulation was non-inferior and had a similar safety profile to the IV dosage form [16]. Additionally, the PrefHer study has reported patient preference for the SC administration for trastuzumab over IV, and that use of SC reduced the time of health care professionals and resources needed to manage patients [20]. This savings in time and cost relative to the IV formulation has been shown in several European studies [21]-[24]. In a multi-centre prospective, observational time and motion study, Burcombe and colleagues investigated the time used for treatment with IV and SC trastuzumab for 24 patients in four centres in England and Wales. The study found that the mean duration for preparation and administration of IV and SC trastuzumab was 94.5 and 24.6 minutes respectively. This would translate into cost savings for the National Health Service (NHS) of $£ 2013$ per patient over one year of treatment with SC trastuzumab [21]. Lastly, a time-andmotion sub-analysis of the PrefHer trial reported time savings of pharmacy and drug administration time between 30\% (in Switzerland) to 51\% (in Spain) from the SC trastuzumab in the SID form compared to IV [24].

Other studies in Europe reported similar findings with the use of SC trastuzumab. Two studies from England and Greece used a modelling-based approach to simulate the time and cost savings [22] [23]. Samanta and colleagues investigated the cost consequences of switching a cohort of 200 breast cancer patients from IV to SC trastuzumab in the English NHS. The study estimated that over one year, switching to SC trastuzumab would result in a reduction of pharmacists and nurses workload by 5492 hours and generate cost savings of $£ 271,000$ [22]. Similarly, a study from Greece reported mean total cost per year of IV and SC trastuzumab was €24,163 and €23,042 per patient respectively; with the use of SC resulting in savings of €1121 [23]. Finally, a report from the Scottish Medicines Consortium indicated that switching from IV to SC trastuzumab would generate cost savings for the Scottish NHS of £3454 over one year of treatment arising from reductions in medication and time costs of health care professionals [25].

In Malaysia, trastuzumab is available in the IV dosage form and is recommended for early breast cancer (EBC) patients who are HER2+ undergoing adjuvant chemotherapy [26]-[29]. Bearing in mind that SC could potentially save cost through savings in personnel time and resource use, we conducted this study to investigate the implications of implementing the use of SC trastuzumab. Although cost savings have been shown in the earlier published studies, we expected that impact on costs could be considerably different in Malaysia. The extent of these savings in Malaysia was important to understand due to differences in treatment practices and unit costs between Malaysia and developed countries. In particular, human resource costs, which are well-known to be high in Europe, are generally lower in government-run facilities in the country. From evidence related to another disease area, it has been shown that for haemodialysis (HD) treatment, human resource costs comprised only $18.9 \%$ of the total cost of HD in the Malaysian Ministry of Health (MOH) dialysis centres compared to $28.9 \%$ in the UK NHS [30] [31]. Given this context, we conducted this study to estimate the differences in cost and resource utilisation between the two different administration routes. The approach taken was to perform a costminimisation analysis (CMA) through adaptation of a mathematical model that compares SC trastuzumab (administered via hand-held syringe) to the IV formulation for adjuvant treatment of HER2+ early breast cancer.

\section{Methods}

\subsection{Model Description}

We performed a local adaptation of a mathematical model developed by Roche, Switzerland, the Herceptin cost-minimisation model (version 1.2). The model was adapted with adjustments for differences in practices and costs in the Malaysian MOH. Costs incurred per patient for the full 1 year course of treatment with IV and SC trastuzumab were taken into consideration. This model was previously utilised in two other CMA studies of SC trastuzumab in England and Scotland [22] [25].

The analysis was performed from two perspectives, namely the $\mathrm{MOH}$ and societal perspectives. Analysis 
from the MOH perspective included the cost categories of healthcare professional time cost, drug cost and consumables cost. Analysis from the societal perspective included the same costs identified in the MOH perspective, but also included patient time costs which were measured by the human capital approach.

The analysis time horizon was one year with the study reference year set as 2014, corresponding to the time of data collection. The study was registered with the Malaysian National Medical Research Register (NMRR14-1470-23387). Since the method used relied on healthcare personnel interviews and mathematical modelling, a full ethics review was not required.

\subsection{Data Sources}

Data used to populate the CMA model was obtained from various sources including official statistics, price lists and estimates from 22 healthcare personnel at four $\mathrm{MOH}$ hospitals. General data input categories required and their sources are shown in Table 1.

Information on treatment practices, drugs and consumables were obtained from four participating MOH hospitals, namely: Penang General Hospital, Sarawak General Hospital, Likas Hospital and Sultan Ismail Hospital. All four hospitals were the main public sector cancer treatment centres in their respective states with oncology departments and in-house pharmacy units for cytotoxic drug reconstitution (CDR). Face-to-face discussions were conducted with healthcare personnel involved in the management of patients and administration of trastuzumab in order to understand the processes of drug preparation and patient management at each site and to collect site estimates of resource and time utilisation. These included oncologists, medical officers, pharmacists and nurses. Interviews and site visits were conducted in November and December 2014.

\subsection{Data Input Assumptions and Details}

Several data inputs required assumptions which are described below:

1) Drug cost and utilisation

Both IV and SC trastuzumab treatment were fixed at 17 cycles based on the current practice of IV trastuzumab administration in the Malaysian MOH. It was assumed that SC would similarly be administered over 17 cycles should the new formulation become available.

Drug dosing during each treatment cycle was assumed to be based on the manufacturer's recommendation. IV treatment would consist of a loading dose $(8 \mathrm{mg} / \mathrm{kg})$ and 16 subsequent doses $(6 \mathrm{mg} / \mathrm{kg})$ while SC treatment would at a dose of $600 \mathrm{mg}$ [17] [18] [32]. As IV dosing was weight-dependent, anonymous data on the body weight of 80 EBC patients treated with trastuzumab obtained from the study sites, comprising 20 patients who were last treated at each site. Unit cost of drugs was based on the manufacturer's indicative price to the MOH for IV (440 mg) and SC (600 mg) trastuzumab. Lastly, based on observation of practices in the CDR pharmacy units at each site, we assumed zero wastage of leftover IV trastuzumab as unmixed IV trastuzumab would be used for the preparation of the next patient's dose.

2) Consumables cost

The cost of consumables was averaged across the unit cost and quantities of resources used at the four study

Table 1. Data input categories and sources.

\begin{tabular}{ll}
\hline Data Input & Input Value and Source \\
\hline Number of cycles of trastuzumab administration over one year & 17 cycles (Assumption based on practice at the participating sites) \\
Consumables utilisation and unit costs & Site estimate \\
Drug doses and unit costs & Roche (Malaysia), drug package insert [17] [18] [32] \\
Usage of leftover IV drugs & $\begin{array}{l}\text { All leftover IV trastuzumab is shared and used (Assumption based on } \\
\text { practice at the participating sites) }\end{array}$ \\
Human resource utilisation (average working hours) & Site estimate \\
Unit cost of human resources & Government salary scales [33] \\
Anonymous patient weights & Data from study sites \\
Patient treatment time & Site estimate \\
Median female wage rate & RM8.22 per hour [34] \\
\hline
\end{tabular}


sites. Consumables included in the analysis were central venous access devices (CVAD) also known as chemoports, items used for drug administration and for IV drug reconstitution. CVAD costs also factored in the proportion of patients who received such devices, which averaged $11.7 \%$ of patients in the study sites. Consumables use for SC administration was based on information from Penang General Hospital as the staff from the other three sites included in this study did not have experience using SC trastuzumab.

3) Health care professional time cost

The cost of health care professionals' time for medical consultation, drug preparation by pharmacists and drug administration activities by nurses were estimated from the time spent for these activities multiplied by the wage rate.

The duration spent on treatment tasks were based on estimated usual practice obtained through interviews with 22 personnel at the four study sites and averaged across the four sites. Healthcare professional time for IV administration was estimated by averaging the duration reported by nurses' at all four sites for patient preparation, drug administration and post-administration tasks. The time spent by the nurse with each patient was calculated by dividing the time for each activity with the number patients under the care of each nurse.

Since SC trastuzumab is not yet available, experience with SC administration was limited to one site (Hospital Penang) with clinical trial experience. This site provided estimates for time needed to prepare and administer SC.

The unit cost of personnel time were estimated from the wage rates of $\mathrm{MOH}$ staff for work performed during usual work hours and included base salaries and fixed allowances.

4) Patient time cost

Patient time cost consisted of the amount of time spent by patients in the oncology day-care unit as estimated by personnel at the study sites. Travel time to reach the hospital was not included in the present analysis. The unit cost of the patients' time was calculated by the human capital approach i.e. estimated using the latest available (year 2012) median female wage rate in Malaysia [34].

\subsection{Analysis}

Base-case analysis was performed by calculating the cost of a full course of treatment (17 cycles over 1 year). The study reference year was fixed at year 2014 as prices used in the study were obtained in late 2014. No discounting of future costs was applied as the treatment duration did not exceed 1 year. Costs were reported in Malaysian Ringgit (RM) values (USD1 = RM3.495 based on the exchange rate on 31/12/2014) [35].

Sensitivity analyses were performed to determine the robustness of the base-case analysis. One-way sensitivity analyses were performed by varying data points of key variables individually. The sensitivity input values are shown in Table 2.

\section{Results}

\subsection{Cost}

The full course of 17 cycles of treatment with IV and SC trastuzumab were estimated to cost RM87154 and

Table 2. One-way sensitivity analyses input values.

\begin{tabular}{lll}
\hline Input & Sensitivity Values (low-high) & Source \\
\hline Patient weight & $55.2-61.1 \mathrm{~kg}$ & Patient weights in study sites \\
Proportion of patients receiving CVAD & $0 \%-95 \%$ & High: Experience of Penang General Hospital Low: Assumption \\
CVAD device cost & $700-1000$ & Estimates from study sites \\
CVAD insertion procedure cost & $263-421$ & Estimates from study sites \\
IV drug administration cost & $596-996$ & Estimates from study sites \\
SC drug administration cost & $148-426$ & Estimates from study sites \\
IV reconstitution (pharmacy) cost & $283-584$ & Estimates from study sites \\
IV patient time cost & $288-1420$ & Estimates from study sites \\
SC patient time cost & $144-366$ & Estimates from study sites \\
\hline
\end{tabular}

Note: ${ }^{*}$ Applicable to analysis from the societal perspective only. 
RM79593 per patient respectively from an MOH perspective, as shown in Figure 1. Aside from drug cost, the major contributors to $\mathrm{MOH}$ costs were consumables and health care professionals' time spent on drug administration ( $0.8 \%$ of IV cost, $0.3 \%$ of SC cost) and IV drug preparation time and consumables used by the CDR pharmacy $(0.5 \%$ of IV cost). Treatment using SC trastuzumab would result in cost savings to the MOH of RM7561 per patient compared to IV. From a societal perspective, the cost of IV and SC trastuzumab was RM87627 and RM79806 per patient respectively, with patient time costs making up $0.5 \%$ of IV cost and $0.3 \%$ of SC cost. Use of SC trastuzumab would generate cost savings to society of RM7820 per patient as shown in Figure 2.

Sensitivity analysis from the MOH perspective indicated a range of cost savings on SC from RM3715 to RM11408 per patient as shown in the tornado diagram in Figure 3, while analysis from the societal perspective indicated a range of cost savings of RM3973 to RM11667 per patient as shown in Figure 4. From both analysis perspectives, cost was most sensitive to assumptions regarding the weight of patients, which effects the cost of IV drugs as IV dosing is weight dependent. Other factors that influenced the results were proportion of patients receiving a CVAD, IV and SC drug administration costs, IV reconstitution costs, cost of the CVAD device and the cost of CVAD insertion procedure. In the societal perspective, patient time costs on IV and SC also had some impact on results.

\section{Time for Treatment Activities}

The estimated time spent for tasks related to treatment with trastuzumab are as shown in Table 3. Specialists would spend an average of 42 minutes per initial patient consultation while medical officers took an average of 24 minutes during each follow-up treatment visit. The consultation time for IV and SC treatment was equal as the choice of formulation would not influence consultation time. IV drug preparation in the pharmacy CDR involved several tasks and required 43.5 minutes of pharmacists' time to prepare an infusion specifically for each patient. In contrast, infusion preparation is not required for the SC formulation, which is a fixed dose and can be prepared for administration by a nurse in the day care unit.

The use of SC trastuzumab would require less nurses' time than the IV formulation. The overall time spent by nurses attending to patients was estimated to be 43.5 minutes for the administration of loading dose and 36.8

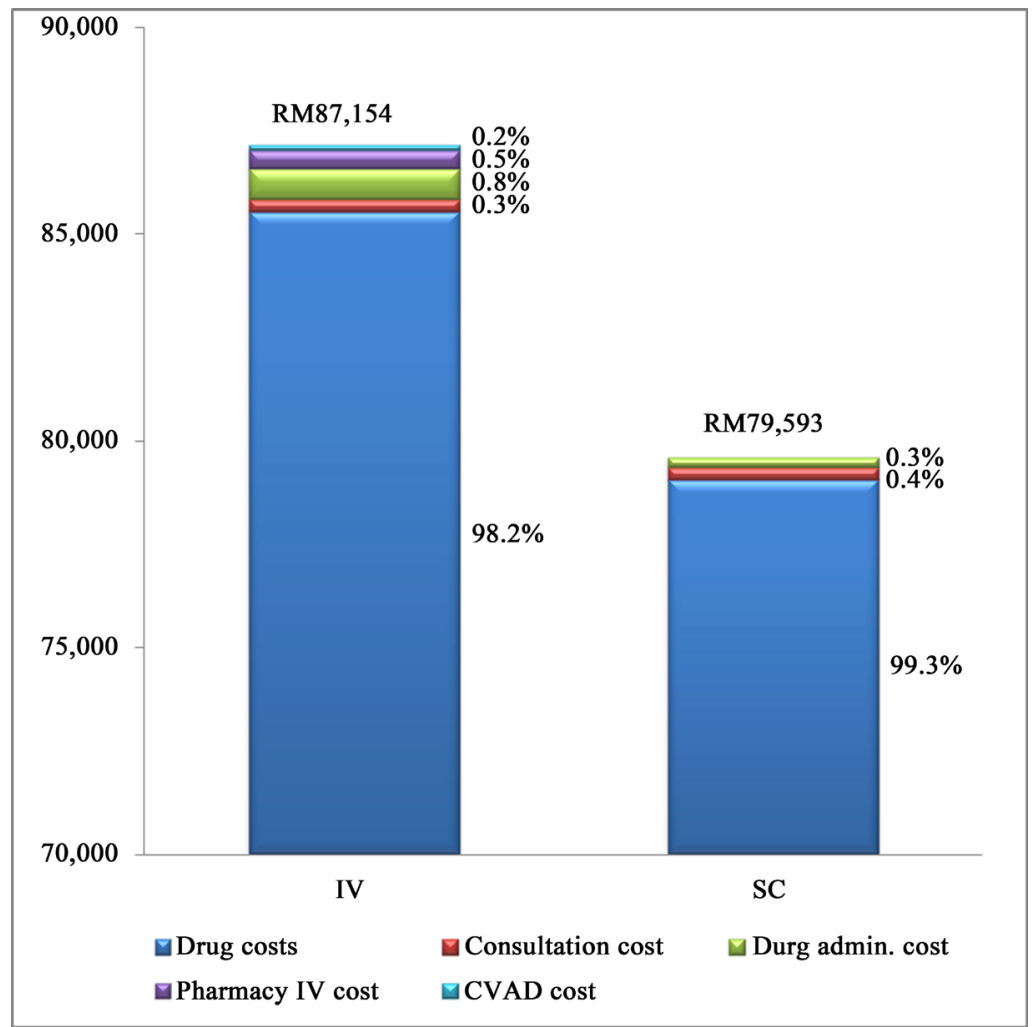

Figure 1. Cost of IV and SC trastuzumab treatment from MOH perspective (RM per patient per year). 


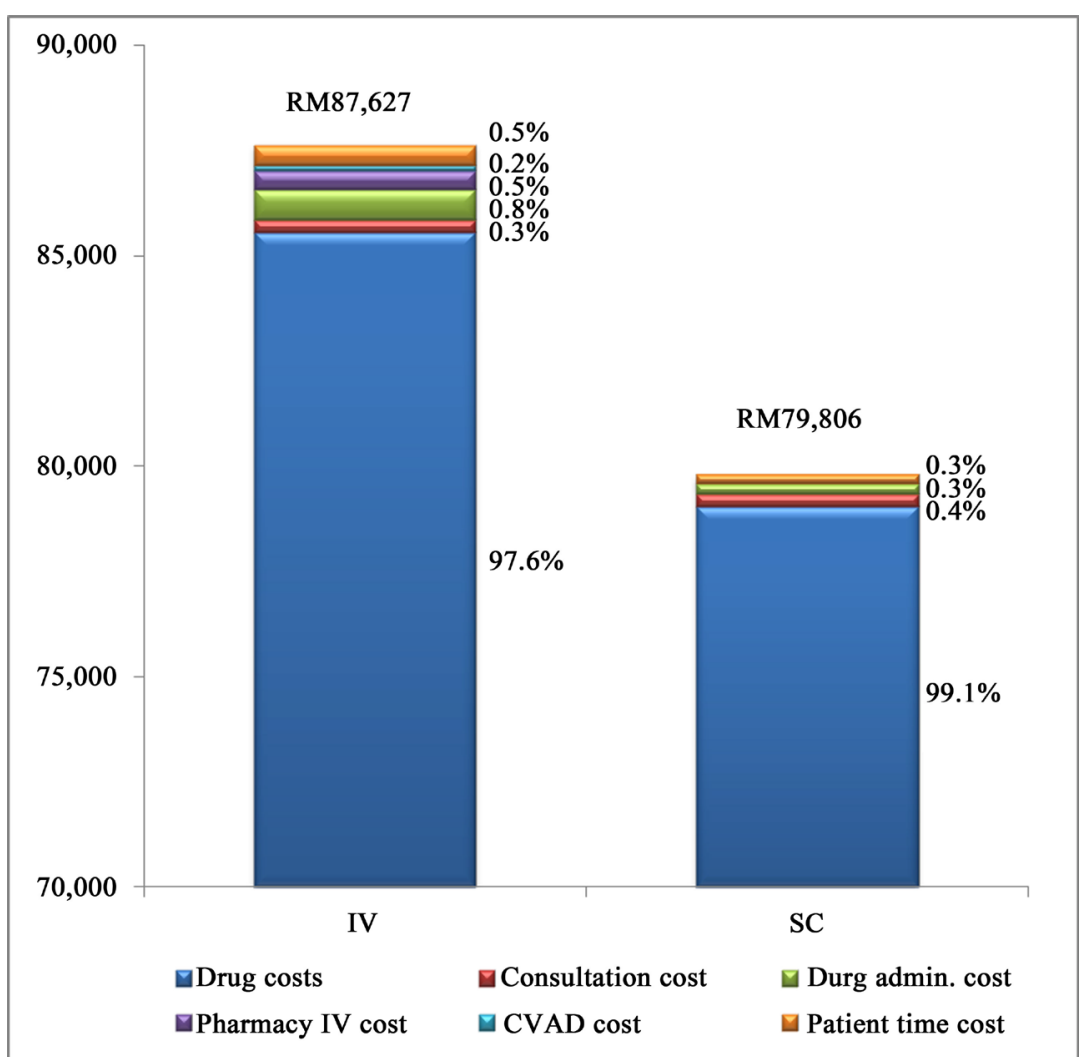

Figure 2. Cost of IV and SC trastuzumab treatment from societal perspective (RM per patient per year).

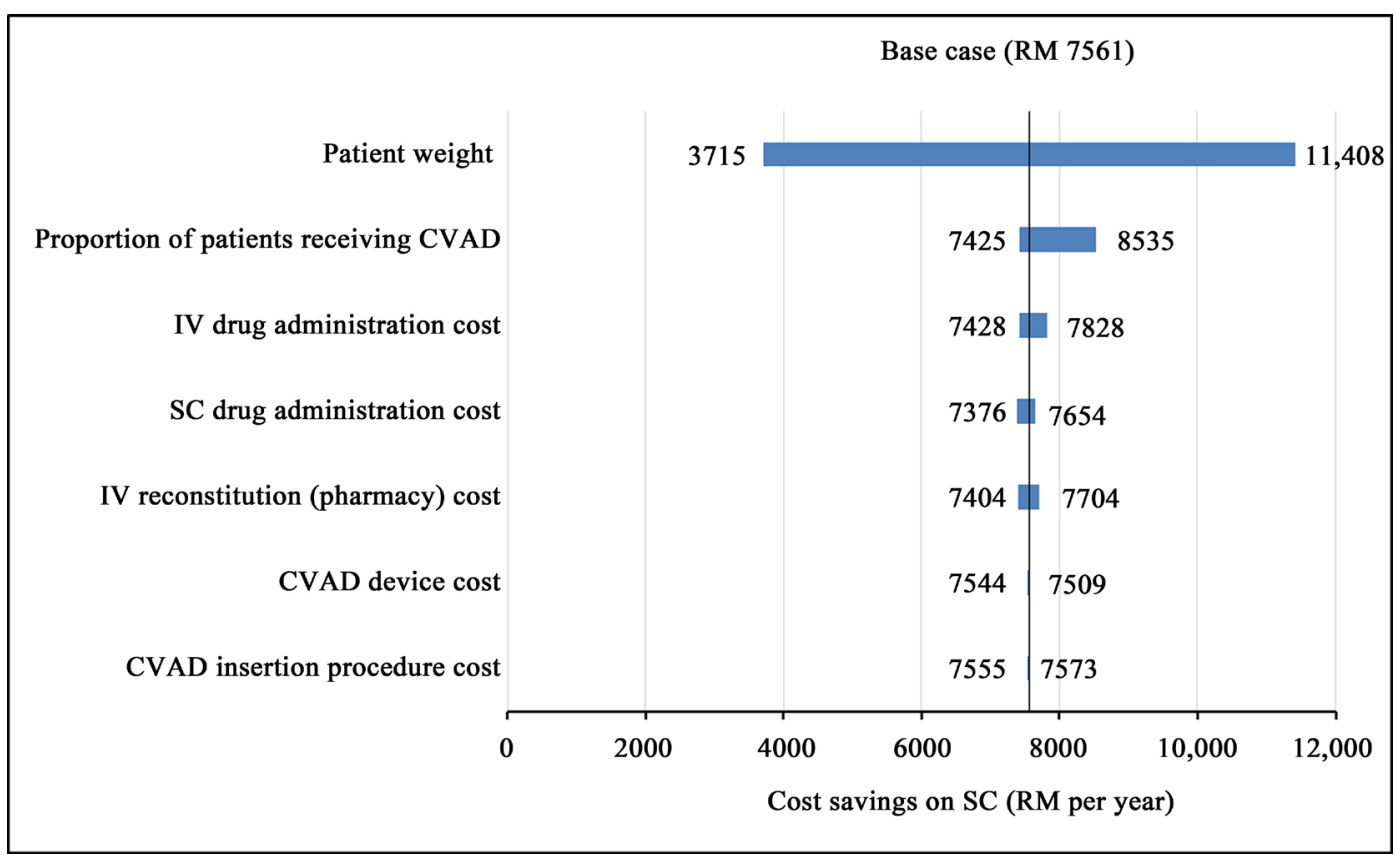

Figure 3. Tornado diagram of one-way sensitivity analyses- $\mathrm{MOH}$ perspective.

minutes for subsequent doses (Table 3) compared to 31.9 minutes using SC, translating to a time saving of 11.6 minutes and 4.9 minutes, respectively. Within this overall time, nurse preparation time for IV took 9.3 minutes for both loading and subsequent doses whereas with SC administration it was estimated to take 5.7 minutes on 


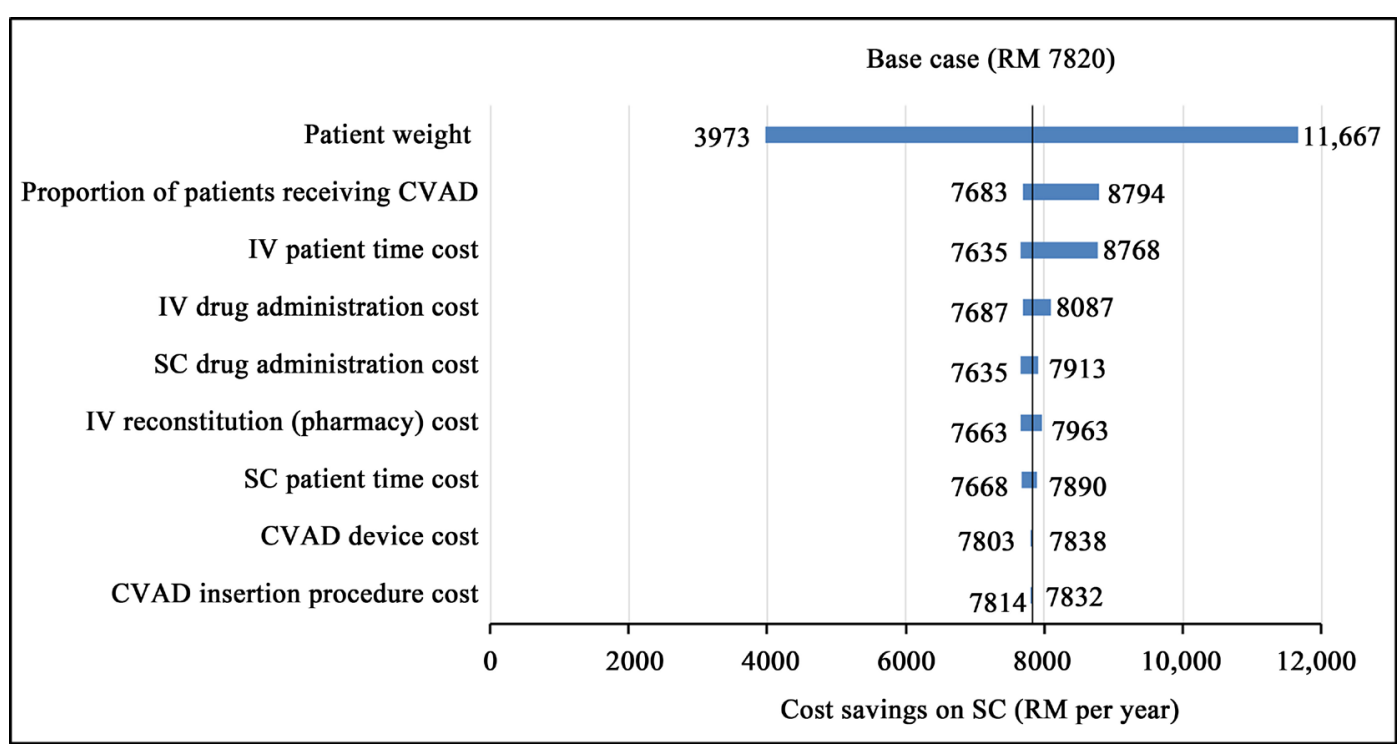

Figure 4. Tornado diagram of one-way sensitivity analyses—-societal perspective.

Table 3. Personnel time by activity (minutes).

\begin{tabular}{|c|c|c|c|c|}
\hline Activity & IV (LD) & IV (SD) & SC & Description \\
\hline \multicolumn{5}{|c|}{ Medical Consultation (minutes per consultation) } \\
\hline Specialist Consultation & 42 & N/A & 42 & $\begin{array}{l}\text { Average duration across study sites for each new patient } \\
\text { consultation with a specialist. }\end{array}$ \\
\hline Medical Officer Consultation & N/A & 24 & 24 & $\begin{array}{l}\text { Average duration across study sites for each follow-up } \\
\text { consultation by a medical officer. }\end{array}$ \\
\hline \multicolumn{5}{|c|}{ CDR Pharmacy Activities (minutes per infusion prepared) } \\
\hline Worksheet and component preparation & 13 & 13 & N/A & $\begin{array}{l}\text { The duration is an average across study sites for each infusion } \\
\text { prepared. }\end{array}$ \\
\hline Isolator room preparation and cleaning & 1 & 1 & N/A & $\begin{array}{l}\text { The duration is an average across study sites. The total time } \\
\text { spent on the task by all pharmacy staff in the isolator room was }\end{array}$ \\
\hline Infusion preparation & 22.5 & 22.5 & N/A & aggregated, then apportioned to each infusion prepared. \\
\hline QA and checking & 7 & 7 & N/A & The duration is an average across study sites. \\
\hline Total CDR pharmacy time & 43.5 & 43.5 & N/A & \\
\hline \multicolumn{5}{|c|}{ Drug Administration Activities (minutes per treatment) } \\
\hline Preparation activities & 9.3 & 9.3 & 5.7 & $\begin{array}{l}\text { Includes tasks for drug collection, drug preparation and patient } \\
\text { preparation. Average of estimated duration across study sites, } \\
\text { then apportioned to each patient under a nurse's care. }\end{array}$ \\
\hline Administration of loading dose & 20 & 13.3 & 6 & $\begin{array}{l}\text { IV dosing duration is based on the manufacturer's prescribing } \\
\text { information [18]. SC dosing duration was estimated by nurses } \\
\text { from Penang General Hospital. Total dosing time is apportioned } \\
\text { to each patient under a nurse's care. }\end{array}$ \\
\hline Post-administration activities & 14.2 & 14.2 & 14.2 & $\begin{array}{l}\text { Includes tasks for saline flushing, IV device removal, patient } \\
\text { monitoring, and scheduling the next appointments. IV duration } \\
\text { was an average of estimated duration across study sites, } \\
\text { apportioned to each patient under a nurse's care. } \\
\text { SC duration is assumed to be equal to IV. }\end{array}$ \\
\hline Total nurse time & 43.5 & 36.8 & 31.9 & \\
\hline
\end{tabular}

Note: $\mathrm{LD}=$ Loading dose; SD = Subsequent dose; CDR activities are not applicable to SC trastuzumab since it does not require reconstitution.

average. In terms of the administering the treatment, IV infusion would take 20 minutes of nurses' time for the loading dose and 13.3 minutes for subsequent doses. With the SC formulation, it would take 6 minutes. The time 
required for post-administration tasks was estimated to be equal for both IV and SC.

\section{Discussion}

Consistent with past studies, we found that the SC formulation of trastuzumab is cost saving compared to the IV formulation currently available in Malaysian MOH hospitals for the treatment of HER2+ early breast cancer [22] [23] [25]. Cost savings for a full course 17 cycles of treatment is estimated to be RM7561 from the MOH perspective to RM7820 from the societal perspective. The analysis indicates that cost savings were mostly derived from savings from drug costs, due to the switch to fixed dose SC dosing from weight-based IV dosing. An examination of the time spent on tasks related to treatment also indicates substantial savings in work duration of healthcare personnel from a switch to SC administration. This was true in terms of nursing time for drug administration but especially so with time spent preparing the IV infusion by CDR pharmacy staff. All one-way sensitivity analyses indicate that the base case findings were robust as SC remained cost saving throughout all variations of input values in both the $\mathrm{MOH}$ and societal analyses.

Our findings of the cost savings of SC are consistent with the results of similar CMA studies from Europe comparing IV and SC trastuzumab. CMAs conducted from the perspective of the England and Wales NHS (£2013), the Greek health system (€1121), and the Scottish NHS (£3454) have all reported cost savings over 1 year of treatment with SC trastuzumab [21] [23] [25]. In addition, an analysis by Samanta et al. estimated cost savings of £271,000 and reduction of pharmacists and nurses workload by 5492 hours from switching a cohort of 200 breast cancer patients from IV to SC trastuzumab in the English NHS [22]. However, the magnitude of cost savings related to personnel costs seen in the European studies are not as apparent in our study due to the comparatively low salaries of $\mathrm{MOH}$ staff.

There were several other benefits of SC which were not incorporated into this study. Since this was a cost-minimisation analysis, other potential impacts were not relevant to the question and had not been included in the model. Yet, these external benefits could be highly impactful. From the perspective of the hospital, SC administration would open uppersonnel and treatment beds (IV infusion stations) currently occupied by patients receiving IV trastuzumab to be reassigned for treatment of other cancer patients. Clearly, benefits in terms of widening access to treatment for other cancers were not within the scope of the present study. There were less tangible benefits that were also not included in the analysis such as the patient's preference. As has been shown by the PrefHer study, if patients were given the choice, they would tend to prefer the SC formulation [20]. Although this was not assessed in this study, the convenience of SC could have impact on patients' satisfaction with the treatment and could increase treatment adherence. Studies in other countries have shown a net positive benefit on Quality Adjusted Life Years [11]-[14]. As well, the use of SC administration in combination with the use of telemedicine may open the pathways to treatment to wider geographical locations when considering the scarcity of clinical oncologists across the country especially in the rural areas. As is the case in two of the centres in this study, Likas Hospital and Sarawak General Hospital, whose patients tend to undertake long journeys from rural locations to obtain treatment due to the geographical spread of their respective states. These various aspects were not part of the scope of our study and may be a thought for future studies and should perhaps also be considered in overall decision-making.

We believe that this study has several strengths and acknowledge its weaknesses. Our use of local data reflective of usual practices in $\mathrm{MOH}$ hospitals can be considered a strength of the study. The time and resource use estimates were derived from data obtained at four MOH hospitals where treatment with IV trastuzumab is routinely provided. The salaries of relevant personnel categories were based on average government salary scales for different staff categories. The time spent by each category of personnel in providing care was a major element of estimation involved in the calculation of non-drug costs. On the other hand, the estimations are averaged across four study sites with different practices and processes. For instance, at one of the sites, reconstitution of drugs was performed on an individual order basis while in the other three centres, reconstitution was done in a batch processing manner in which all IV prescription orders for the day were obtained before reconstitution was begun. The variation in practices at different sites can be considered another advantage of the study since it provided a strong basis on which to conduct the sensitivity analysis. Overall, despite some challenges in the way we calculated the cost and resource use, we believe that the result can provide insight into the cost of IV and SC treatment at $\mathrm{MOH}$ centres.

On the other hand, it could be argued that there are certain weaknesses in our approach. As mentioned, we relied on estimates and opinions of the study investigators to estimate data inputs. Although we had involved four 
study sites and averaged the time and resource use to limit bias, since the method was based on opinion and recall rather than direct measurement of time expended in each activity as was performed in the study by Burcombe, there could still be potential information bias in the estimation [21]. However, the estimated nurses time for IV drug administration in this study (20 and 13.3 minutes for loading and subsequent doses) was within the range of those reported in other studies which ranged from 2 minutes in Denmark and Spain to 35 minutes in the UK per IV administration, and up to 60 minutes for an IV loading dose in the National University Hospital of Iceland [21] [24] [36].

Furthermore, since we observed differences in practices at these four sites, it is possible that other MOH sites have other variations in their practice patterns which would limit generalisability to other sites. It should also be pointed out that the estimates of SC consumables cost and personnel time are likely weaker than the estimates for IV due to a lack of experience with SC trastuzumab. The only site with experience of SC administration was the Penang General Hospital, and their experience was limited to a clinical trial which may not reflect future routine clinical practice. Finally, it is arguable that a model provided by the study sponsor could give rise to doubts about its structure or content. However, this cost-minimisation model was fairly simple and straightforward with the results being driven by data inputs provided by the team of author-investigators and study sites.

There are other limitations related to assumptions used in the study which should perhaps be noted but are unlikely to have any bearing on the results. Firstly, we did not consider costs of treating drug related adverse events. Since the study compared two formulations of the same drug which has been shown to have no statistically significant differences in serious adverse events, the costs of treating adverse events were assumed to be equal between IV and SC and should not alter the conclusion of cost savings of SC [16]. Secondly, the extent to which the national median female wage rate accurately reflects the opportunity cost of patient time can also be considered a weakness but this was applied equally to both sides of the model. Thirdly, in the societal perspective, the study did not estimate the cost of carer's time even though it is quite common for Malaysian patients to be accompanied by a relative for their treatment. If this had been included, the results would strengthen the cost of SC relative to that of IV treatment. Finally, we note that a small proportion of patients on IV trastuzumab were assumed to have CVAD insertions. According to local practices, CVADs are not routinely inserted for other chemotherapy but would be considered for IV trastuzumab patients due to the number of cycles needed to complete treatment. When it is used, the CVAD devices may increase the risk of CVAD-related adverse events which was not taken into account. As CVAD devices are only used in IV drug administration, the exclusion of costs related to CVAD-related adverse events would tend to favour the IV formulation. Overall, the approach we used in this study can at best be considered conservative and at worst be considered neutral in its assessment of SC use.

We believe that our study provides useful evidence about SC trastuzumab given unavoidable economic constraints. Regardless of the healthcare system in place, the question of value for money is a constant and good decision-making becomes paramount. We acknowledge that there are several potential benefits of SC trastuzumab that we could not address within the scope of this study which we had intended to keep as a simple comparison of two choices by using the cost-minimization method. Both choices are in fact equally good since they are based on the same treatment, with the major difference being the method of administration and added cost-savings through the standard dose per SC injection.

\section{Conclusion}

Malaysia is faced with a significant challenge to both prevent the occurrence of new cases while at the same time extending the lives of women who have confirmed breast cancer [1] [7] [8]. The increasing mortality rates add another layer of complexity to be overcome. Our small contribution to the current body of knowledge does not provide all the answers to the important questions of how to achieve this grand vision. Nonetheless, thus far, too little published evidence has addressed this issue in Malaysia. To the best of our knowledge, this is a first study to assess the issue of cost to the Malaysian $\mathrm{MOH}$ in breast cancer treatment while specifically investigating the cost of IV and SC trastuzumab. Both the $\mathrm{MOH}$ and societal perspectives were assessed and the use of the SC formulation was found to be advantageous. Despite some limitations as stated above, we believe that the study provides useful insights into the potential impact on costs if the SC formulation was used in place of IV infusion. Furthermore, the use of SC formulation has the potential to improve overall efficiency of oncology units and enable more patients to receive treatment. This could be the basis of a future study once the SC for- 
mulation is in use. Given the significant issues around cancer care faced by the nation among other competing priorities, the SC formulation has many favourable aspects. Our findings provide a balance of evidence in favour of SC trastuzumab use in place of the IV formulation.

\section{Acknowledgements}

This study was sponsored by Roche (Malaysia) Sdn Bhd. Goh A and Azmi S are employees of Veras Research who were paid by Roche to develop this study. The authors thank Clement Tournier of Roche for providing the Herceptin CMA 1.2 model to be used in the adaptation; and the doctors, pharmacists, day care nurses and medical assistants from Sultan Ismail Hospital, Likas Hospital, Penang General Hospital, and Sarawak General Hospital for their cooperation with the site visits. Lastly, we thank the Director-General of the Ministry of Health, Malaysia for permission to publish this paper.

\section{References}

[1] Youlden, D.R., Cramb, S.M., Yip, C.H. and Baade, P.D. (2014) Incidence and Mortality of Female Breast Cancer in the Asia-Pacific Region. Cancer Biology \& Medicine, 11, 101-115.

[2] Yip, C.H., Cazap, E., Anderson, B.O., Bright, K.L., Caleffi, M., et al. (2011) Breast Cancer Management in MiddleResource Countries (MRCs): Consensus Statement from the Breast Health Global Initiative. Breast, 20, S12-S19. http://www.ncbi.nlm.nih.gov/pubmed/21388811 http://dx.doi.org/10.1016/j.breast.2011.02.015

[3] Bhoo-Pathy, N., Hartman, M., Yip, C.H., Saxena, N., Taib, N.A., et al. (2012) Ethnic Differences in Survival after Breast Cancer in South East Asia. PLoS One, 7, e30995. http://dx.doi.org/10.1371/journal.pone.0030995

[4] Lim, G.C., Aina, E.N., Cheah, S.K., Ismail, F., Ho, G.F., et al. (2014) Closing the Global Cancer Divide-Performance of Breast Cancer Care Services in a Middle Income Developing Country. BMC Cancer, 14, 212. http://dx.doi.org/10.1186/1471-2407-14-212

[5] World Health Organization (2010) Country Cooperation Strategy 2009-2013. http://www.who.int/countryfocus/cooperation_strategy/ccs_mys_en.pdf?ua=1

[6] Merican, M.I., Rohaizat, Y. and Haniza, S. (2004) Developing the Malaysian Health System to Meet the Challenges of the Future. Medical Journal of Malaysia, 84-93. http://www.ncbi.nlm.nih.gov/pubmed/15535341

[7] Das, D. and Win, K. (2011) Breast Cancer-Its Impact on National Resource and Survival. RGMHR, 2. http://www.iomcworld.com/rgmhr/files/004.pdf

[8] Yen, S., Abd Shatar, A. and Hashim, H. (2011) The Socioeconomic Impacts of Breast Cancer on Breast Cancer Patients in Penang. Kajian Malaysia, 29, 111-126. http://web.usm.my/km/29(2)2011/Art6_KM29-2.pdf

[9] Pinto, A.C., Ades, F., de Azambuja, E. and Piccart-Gebhart, M. (2013) Trastuzumab for Patients with HER2 Positive Breast Cancer: Delivery, Duration and Combination Therapies. Breast, 22, S152-S155. http://dx.doi.org/10.1016/j.breast.2013.07.029

[10] Perez, E.A., Romond, E.H., Suman, V.J., Jeong, J.H., Davidson, N.E., et al. (2011) Four-Year Follow-Up of Trastuzumab Plus Adjuvant Chemotherapy for Operable Human Epidermal Growth Factor Receptor 2-Positive Breast Cancer: Joint Analysis of Data From NCCTG N9831 and NSABP B-31. Journal of Clinical Oncology, 29, 3366-3373. http://dx.doi.org/10.1200/JCO.2011.35.0868

[11] Liberato, N.L., Marchetti, M. and Barosi, G. (2007) Cost Effectiveness of Adjuvant Trastuzumab in Human Epidermal Growth Factor Receptor 2-Positive Breast Cancer. Journal of Clinical Oncology, 25, 625-633. http://dx.doi.org/10.1200/JCO.2006.06.4220

[12] Kurian, A.W., Thompson, R.N., Gaw, A.F., Arai, S., Ortiz, R., et al. (2007) A Cost-Effectiveness Analysis of Adjuvant Trastuzumab Regimens in Early HER2/Neu-Positive Breast Cancer. Journal of Clinical Oncology, 25, 634-641. http://dx.doi.org/10.1200/JCO.2006.06.3081

[13] Garrison Jr., L.P., Lubeck, D., Lalla, D., Paton, V., Dueck, A., et al. (2007) Cost-Effectiveness Analysis of Trastuzumab in the Adjuvant Setting for Treatment of HER2-Positive Breast Cancer. Cancer, 110, 489-498. http://dx.doi.org/10.1002/cncr.22806

[14] de Lima Lopes Jr., G. (2011) Societal Costs and Benefits of Treatment with Trastuzumab in Patients with Early HER2neu-Overexpressing Breast Cancer in Singapore. BMC Cancer, 11, 178. http://dx.doi.org/10.1186/1471-2407-11-178

[15] Yip, C.H., Bhoo Pathy, N. and Teo, S.H. (2014) A Review of Breast Cancer Research in Malaysia. The Medical Journal of Malaysia, 69, 8-22. http://www.ncbi.nlm.nih.gov/pubmed/25417947 
[16] Ismael, G., Hegg, R., Muehlbauer, S., Heinzmann, D., Lum, B., et al. (2012) Subcutaneous versus Intravenous Administration of (Neo)adjuvant Trastuzumab in Patients with HER2-Positive, Clinical Stage I-III Breast Cancer (HannaH Study): A Phase 3, Open-Label, Multicentre, Randomised Trial. The Lancet Oncology, 13, 869-878.

http://www.ncbi.nlm.nih.gov/pubmed/22884505

http://dx.doi.org/10.1016/S1470-2045(12)70329-7

[17] Roche (Malaysia). Pack Insert for Malaysia. Herceptin ${ }^{\circledR}$ Trastuzumab. http://www.bpfk.gov.my/Biro/11792-H/610420-05-5584/pharma/MYHerceptin1208cds7.0cv(2).pdf

[18] Genentech (2014) HERCEPTIN ${ }^{\circledR}$ [Trastuzumab] Prescribing Information. http://www.gene.com/download/pdf/herceptin_prescribing.pdf

[19] Roche (2013) CHMP Recommend EU Approval of Roche’s Subcutaneous Herceptin for HER2 Positive Breast Cancer. http://www.roche.com/media/store/releases/med-cor-2013-06-28.htm

[20] Pivot, X., Gligorov, J., Muller, V., Curigliano, G., Knoop, A., et al. (2014) Patients’ Preferences for Subcutaneous Trastuzumab versus Conventional Intravenous Infusion for the Adjuvant Treatment of HER2-Positive Early Breast Cancer: Final Analysis of 488 Patients in the International, Randomized, Two-Cohort PrefHer Study. Annals of Oncology, 25, 1979-1987. http://dx.doi.org/10.1093/annonc/mdu364

[21] Burcombe, R., Chan, S., Simcock, R., Samanta, K. and Percival, F. (2013) Subcutaneous Trastuzumab (Herceptin ${ }^{\circledR}$ ): A UK Time and Motion Study in Comparison with Intravenous Formulation for the Treatment of Patients with HER2-Positive Early Breast Cancer. Advances in Breast Cancer Research, 2, 133-140. http://dx.doi.org/10.4236/abcr.2013.24022

[22] Samanta, K., Moore, L., Jones, G., Evason, J. and Owen, G. (2012) Potential Time and Cost Savings with Herceptin (trastuzumab) Subcutaneous (SC) Injection versus Herceptin Intravenous (IV) Infusion: Results from Three Different English Patient Settings. ISPOR 15th Annual European Congress, Berlin, 3-7 November 2012. http://www.ispor.org/research pdfs/42/pdffiles/PCN39.pdf

[23] Mylonas, C., Kourlaba, G., Fountzilas, G., Skroumpelos, A. and Maniadakis, N. (2014) Cost-Minimization Analysis of Trastuzumab Intravenous versus Trastuzumab Subcutaneous for the Treatment of Patients with HER2+ Early Breast Cancer and Metastatic Breast Cancer in Greece. Value in Health, 17, A640-A641. http://dx.doi.org/10.1016/j.jval.2014.08.2310

[24] De Cock, E., Semiglazov, V., Lopez-Vivanco, G., Verma, S., Pivot, X., et al. (2013) Time Savings with Trastuzumab Subcutaneous vs. Intravenous Administration: A Time and Motion Study. 13th St. Gallen International Breast Cancer Conference, St. Gallen, 13-16 March 2013.

[25] Scottish Medicines Consortium (2013) Trastuzumab, 600mg/5mL Solution for Injection (Herceptin ${ }^{\circledR}$ ). SMC No. (928/13). https://www.scottishmedicines.org.uk/files/advice/trastuzumab Herceptin FINAL December 2013 for website.pdf

[26] Ministry of Health Malaysia (2002) Clinical Practice Guidelines: Management of Breast Cancer. MOH/P/PAK/212.10 (GU). http://www.acadmed.org.my/view_file.cfm?fileid=194

[27] Ministry of Health Malaysia (2015) Ministry of Health Drug Formulary. http://www.pharmacy.gov.my/v2/sites/default/files/document-upload/pfukkm-2-2015-jun.pdf

[28] Goldhirsch, A., Winer, E.P., Coates, A.S., Gelber, R.D., Piccart-Gebhart, M., et al. (2013) Personalizing the Treatment of Women with Early Breast Cancer: Highlights of the St Gallen International Expert Consensus on the Primary Therapy of Early Breast Cancer 2013. Annals of Oncology, 24, 2206-2223. http://dx.doi.org/10.1093/annonc/mdt303

[29] Senkus, E., Kyriakides, S., Penault-Llorca, F., Poortmans, P., Thompson, A., et al. (2013) Primary Breast Cancer: ESMO Clinical Practice Guidelines for Diagnosis, Treatment and Follow-Up. Annals of Oncology, 24, vi7-vi23. http://dx.doi.org/10.1093/annonc/mdt284

[30] Hooi, L.S., Lim, T.O., Goh, A., Wong, H.S., Tan, C.C., et al. (2005) Economic Evaluation of Centre Haemodialysis and Continuous Ambulatory Peritoneal Dialysis in Ministry of Health Hospitals, Malaysia. Nephrology, 10, 25-32. http://dx.doi.org/10.1111/j.1440-1797.2005.00360.x

[31] Baboolal, K., McEwan, P., Sondhi, S., Spiewanowski, P., Wechowski, J., et al. (2008) The Cost of Renal Dialysis in a UK Setting-A Multicentre Study. Nephrology Dialysis Transplantation, 23, 1982-1989. http://dx.doi.org/10.1093/ndt/gfm870

[32] Leveque, D. (2014) Subcutaneous Administration of Anticancer Agents. Anticancer Research, 34, 1579-1586. http://www.ncbi.nlm.nih.gov/pubmed/24692685

[33] Jabatan Perkhidmatan Awam (2013) Penambahbaikan Jadual Gaji Minimum-Maksimum Gred 1 Hingga Gred 54 Di Bawah Sistem Saraan Malaysia. http://docs.jpa.gov.my/docs/pp/2013/pp362013.pdf

[34] Department of Statistics Malaysia (2013) Salaries and Wages Survey Report: Malaysia 2012. https://newss.statistics.gov.my/newss-portalx/ep/epFreeDownloadContentSearch.seam?contentId=24728\&actionMetho d=ep\%2FepFreeDownloadContentSearch.xhtml\%3AcontentAction.doDisplayContent\&cid=27121 
[35] Bank Negara Malaysia (2015) Exchange Rates.

http://www.bnm.gov.my/index.php?ch=statistic\&pg=stats_exchangerates

[36] Agnarsson, S. and Jónsdóttir, H. (2013) Comparison of Costs of Different Methods of Administering Herceptin (Trastuzumab). Institute of Economic Studies, University of Iceland, Reykjavík.

http://hhi.hi.is/sites/hhi.hi.is/files/C-Series/2013/C13 04.pdf 